\title{
Gravitino dark matter with constraints from Higgs boson mass and sneutrino decays
}

\author{
Leszek Roszkowski, ${ }^{a, 1}$ Sebastian Trojanowski, ${ }^{a}$ Krzysztof Turzyński $^{b}$ and \\ Karsten Jedamzik ${ }^{c}$ \\ ${ }^{a}$ National Centre for Nuclear Research, \\ Hoża 69, 00-681, Warsaw, Poland \\ ${ }^{b}$ Institute of Theoretical Physics, Faculty of Physics, University of Warsaw, \\ Hoża 69, 00-681, Warsaw, Poland \\ ${ }^{c}$ Laboratoire de Physique Theorique et Astroparticules, \\ UMR520\%-CRNS, Université Montpellier II, \\ 34095 Montpellier, France \\ E-mail: L.Roszkowski@sheffield.ac.uk, \\ Sebastian.Trojanowski@fuw.edu.pl, \\ Krzysztof-Jan.Turzynski@fuw.edu.pl, karsten.jedamzik@univ-montp2.fr
}

ABSTRACT: We investigate gravitino dark matter produced thermally at high temperatures and in decays of a long-lived sneutrino. We consider the Non-Universal Higgs Model and a generalized gauge mediation model, and in each case identify sneutrino LOSP regions of the parameter space consistent with the mass of the Higgs-like boson observed at the LHC. We apply relevant collider and cosmological bounds, including constraints from Big Bang Nucleosynthesis and from warm dark matter on large scale structures. Generally, we find allowed values of the reheating temperature $T_{R}$ below $10^{9} \mathrm{GeV}$, i.e. somewhat smaller than the values needed for thermal leptogenesis, even with a conservative lower bound of $122 \mathrm{GeV}$ on the Higgs mass. Requiring mass values closer to $126 \mathrm{GeV}$ implies $T_{R}$ below $10^{7} \mathrm{GeV}$ and the gravitino mass less than $10 \mathrm{GeV}$.

KeYwords: Supersymmetry Phenomenology

ARXiv EPRINT: 1212.5587

\footnotetext{
${ }^{1}$ On leave of absence from the University of Sheffield, U.K.
} 


\section{Contents}

1 Introduction 1

2 Review of constraints in sneutrino LOSP scenarios 3

2.1 Soft supersymmetry breaking masses at the low scale 3

2.2 Conditions for $m_{L}^{2}<m_{E}^{2}$ from renormalization group running from a high scale

2.3 Higgs boson mass of $126 \mathrm{GeV}$

2.4 Bound on the reheating temperature from BBN 7

$\begin{array}{lll}3 & \text { Numerical analysis } & 7\end{array}$

3.1 The NUHM 8

$\begin{array}{lll}3.2 & \text { GGM models } & 15\end{array}$

4 Conclusions $\quad 17$

A Soft supersymmetry breaking parameters at the high scale 18

\section{Introduction}

Of all extensions of the Standard Model of elementary particles, its supersymmetric version (Minimal Supersymmetric Standard Model, MSSM) still remains particularly well motivated (for a review, see, e.g., [1]). Several mechanisms have been proposed to describe the origin and mediation of the necessary supersymmetry breaking in the MSSM, yielding distinctive mass spectra for the supersymmetric partners of the known particles. Among these, models equipped with $R$-parity predict that the lightest supersymmetric particle (LSP) is stable which allows for a possibility that it constitutes dark matter (DM) in the Universe.

In the MSSM, the most popular DM particles are: the lightest neutralino, the gravitino (present in the MSSM embedded in supergravity) or the axino (in the MSSM extended with a $\mathrm{U}(1)_{\mathrm{PQ}}$ symmetry) [2]. Different properties of these particles require different variants of the history of the early Universe, including the observationally determined abundance of DM. The lightest neutralino LSP is considered as perhaps the most natural choice for $\mathrm{DM}$ as its relic abundance from thermal freeze-out can agree with observations, $\Omega_{\mathrm{CDM}} h^{2}=$ $0.113 \pm 0.004$ [3], for a $\mathcal{O}(100) \mathrm{GeV}$, although scenarios in which the lightest neutralinos are produced in decays of heavier particles previously dominating the energy density of the Universe, have also been proposed, see, e.g., [4].

The abundance of extremely weakly interacting particles (EWIMPs), like the gravitino or the axino LSP, is determined in a way markedly different from that of the lightest neutralino. They are produced in scatterings of other particles in the primordial plasma; the 
abundance of such thermally produced EWIMP is proportional to the reheating temperature $T_{R}$. In addition to thermal production (TP), EWIMPs can also be produced in the decays of the next-to-lightest supersymmetric particles (NLSP), which are usually the lightest ordinary supersymmetric particles (LOSP). While in general nearly any MSSM particle can be the LOSP, the most natural choices, other than the lightest neutralino, are: the lighter stau (or stop), or the sneutrino. It is this last case that we will consider in this paper.

In non-thermal production (NTP) from LOSP decays, the LOSP lifetime may be long enough for energetic decay products to affect the abundances of the light elements (particularly in the case of gravitinos, while much less so for axinos). A good agreement of predictions of the standard Big Bang Nucleosynthesis (BBN) with observations sets stringent limits on such additional contributions (for reviews, see e.g. [5-7]). The resulting picture can be roughly summarized as follows: the constrains are weakest for light (small energy release in decay), short-lived $(\tau<\mathcal{O}(100 \mathrm{sec}))$ and not too-abundant decaying particles, but above all for decays with a small hadronic branching ratio. Therefore, of all possible LOSPs, sneutrino decays are the least constrained by BBN since hadronic showers can only be produced in (strongly suppressed) 3- and 4-body sneutrino decays. It should also be noted that the present LHC data, albeit quite restrictive for the gluinos and the colored scalar partners of quarks of the first and second generation, still allow sleptons and sneutrinos with much lower masses. All this makes supersymmetric models with gravitino LSP and sneutrino LOSP quite attractive phenomenologically from the bottom-up perspective. ${ }^{1}$

In the simplest scenarios of supersymmetry breaking the sneutrino is not the LOSP and there have been just a handful of studies devoted to analyzing BBN constraints on sneutrino LOSP [9-12] in theoretically motivated scenarios, such as the Non-Universal Higgs Model (NUHM) [13-15] or the Generalized Gauge Mediation (GGM) model [16-19].

In the present letter we re-visit the possibility of gravitino dark matter from both TP and from NTP of sneutrino decays in light of recent discovery at the LHC of a Higgs-like boson with a mass of $126 \mathrm{GeV}[20,21]$. We will show that taking this new result at face value implies a stringent upper bound on the reheating temperature $T_{R}$ below $10^{7} \mathrm{GeV}$, and also favors a low gravitino mass region, below $10 \mathrm{GeV}$. Assuming a conservative lower bound on the Higgs boson mass of $122 \mathrm{GeV}$ leads to weaker constraints on $T_{R}$ and allows a larger gravitino mass.

So far $T_{R}$ has been allowed to take the largest values for the sneutrino LOSP of all MSSM choices owing to the lowest yield at freeze-out. This had a double effect of having the weakest effect on BBN and also requiring largest $T_{R}$ for the TP contribution to make up for the reduced gravitino relic abundance from NTP. However, the relatively large Higgs mass implies larger SUSY breaking mass scale, thus also larger masses of gauginos whose scatterings dominate TP. This in turn boost the TP contribution (which is proportional to their square) and, as a consequence, requires lower $T_{R}$. A larger SUSY breaking scale also implies larger sneutrino mass, and, as a consequence, larger yield at freeze-out and more energetic hadronic showers, which translates to more stringent BBN bounds.

\footnotetext{
${ }^{1}$ The case with light sneutrino LSP, constituting even a small portion of DM, is very strongly constrained by direct detection experiments [8]; in fact, the results of the XENON100 experiment require this contribution to be at most of the order of $10^{-3}$ of a relic sneutrino abundance.
} 
In addition to analyzing the effect of the Higgs mass on the scenario, we extend and update previous analyses in several directions. First, for small and moderate $\tan \beta$ we identify the patterns of soft supersymmetry breaking masses at the high scale that can lead to the sneutrino NLSP. In realistic models, sneutrinos are often degenerate in mass with right-handed sleptons and/or neutralinos (in addition to degeneracy with corresponding left-handed charged sleptons). This leads to many coannihilation channels, which may affect the relic density $\Omega_{\mathrm{NLSP}} h^{2}$ and, as a consequence, also BBN bounds, as they are sensitive to $\Omega_{\mathrm{NLSP}} h^{2}$. Second, we implement the BBN bounds using a state-of-the-art numerical code for solving the relevant Boltzmann equations [22]. As an input parameter to that code, we perform a full computation of the hadronic branching fraction of sneutrino decays, including quark-antiquark production from on-shell and off-shell electroweak gauge bosons and gauginos.

The paper is organized as follows. In section 2, we review the constraints in $\tilde{\nu}$ LOSP scenarios, presenting the assumptions leading to sufficiently light sneutrinos, together with the constraints from the Higgs boson mass measurement and BBN. In section 3, we perform a numerical analysis of $\tilde{\nu}$ LOSP scenarios, discussing the impact of various constraints on the NUHM and the GGM model. We present our conclusions in section 4.

\section{Review of constraints in sneutrino LOSP scenarios}

\subsection{Soft supersymmetry breaking masses at the low scale}

The $\tau$-sneutrino, $\tilde{\nu}_{\tau}$, is the lightest of the sneutrinos due to the $\tau$-Yukawa coupling driving its mass slightly below the sneutrinos of the other two generations, and from now on we will refer to it as simply the sneutrino. The sneutrino can become lighter than its charged slepton partner thanks to the electroweak $D$-term contributions to the slepton and the sneutrino masses. For moderate $\tan \beta$ the sneutrino mass after electroweak symmetry breaking (EWSB) reads (see e.g. [23])

$$
m_{\tilde{\nu}}^{2}=m_{L}^{2}+D_{\tilde{\nu}}^{2}
$$

whereas the mass matrix of the charged sleptons of the third generation is given by

$$
\mathbf{m}_{\tilde{\tau}_{L, R}}^{2}=\left(\begin{array}{cc}
m_{L}^{2}+D_{\tilde{\ell}_{L}}^{2} & m_{\tau}\left(\mu \tan \beta-A_{\tau}\right) \\
m_{\tau}\left(\mu \tan \beta-A_{\tau}\right) & m_{E}^{2}+D_{\tilde{\ell}_{R}}^{2}
\end{array}\right) .
$$

In (2.1) and (2.2) $m_{L}^{2}$ and $m_{E}^{2}$ denote soft supersymmetry breaking mass parameters of the superpartners of the left-handed and the right-handed leptons, respectively, and $A_{\tau}$ stands for the $\tau$ trilinear parameter, with all the parameters evaluated at the EWSB scale. The $D$-term contribution to the sneutrino mass, $D_{\tilde{\nu}}^{2}=-\frac{1}{2} M_{Z}^{2}$, is negative, while analogous contributions to the masses of the charged sleptons, $D_{\tilde{\ell}_{L}}^{2}=M_{W}^{2}-\frac{1}{2} M_{Z}^{2}$ and $D_{\tilde{\ell}_{R}}^{2}=M_{Z}^{2}-M_{W}^{2}$, are positive.

The sneutrino mass (2.1) is smaller than the smaller of the eigenvalues of the slepton mass matrix (2.2) if the condition

$$
m_{E}^{2}-m_{L}^{2}>\frac{m_{\tau}^{2}\left(\mu \tan \beta-A_{\tau}\right)^{2}}{M_{W}^{2}}+M_{W}^{2}-\frac{3}{2} M_{Z}^{2}
$$




\begin{tabular}{|c|ccccc|ccccc|}
\hline$m_{S}$ & $c_{E 1}$ & $c_{L 1}$ & $c_{L 2}$ & $\tilde{c}_{E U}$ & $\tilde{c}_{L Q}$ & $c_{E 1}$ & $c_{L 1}$ & $c_{L 2}$ & $\tilde{c}_{E U}$ & $\tilde{c}_{L Q}$ \\
& \multicolumn{8}{|c|}{$Q=10^{14} \mathrm{GeV}$} & & \multicolumn{5}{c}{$Q=10^{16} \mathrm{GeV}$} & \\
\hline $500 \mathrm{GeV}$ & 0.47 & 0.12 & 0.52 & -0.0027 & -0.0049 & 0.62 & 0.15 & 0.64 & -0.0038 & -0.0060 \\
$1000 \mathrm{GeV}$ & 0.45 & 0.11 & 0.51 & -0.0026 & -0.0048 & 0.59 & 0.15 & 0.62 & -0.0037 & -0.0059 \\
\hline
\end{tabular}

Table 1. Numerical values of the coefficients $c_{E 1}, c_{L 1}, c_{L 2}, \tilde{c}_{E U}, \tilde{c}_{L Q}$ in (2.4) for two representative choices of the high scale $Q$ and of the EWSB mass scale $m_{S}=\sqrt{m_{\tilde{t}_{1}} m_{\tilde{t}_{2}}}$.

is satisfied. For typical values of the parameters, it follows from the inequality (2.3) that the sneutrino is the lightest slepton if $m_{L}^{2}<m_{E}^{2}$ and the left-right mixing in the slepton sector is not too large. For example, with $\mu=1000 \mathrm{GeV}$ and $\tan \beta=10$, the condition (2.3) is satisfied if the splitting between $\sqrt{m_{E}^{2}}$ and $\sqrt{m_{L}^{2}}$ is of at least about $100 \mathrm{GeV}$. It is also clear that increasing $\tan \beta$ while keeping the other parameters in (2.1) and (2.2) fixed decreases the mass of the lighter charged slepton of each generation, eventually closing the region of the parameters where the sneutrino is the lightest slepton.

\subsection{Conditions for $m_{L}^{2}<m_{E}^{2}$ from renormalization group running from a high scale}

Since sneutrino LOSP disfavors large $\tan \beta$, we can, following the method outlined in [24], obtain solutions of renormalization group equations (RGEs) for the soft supersymmetry breaking parameters $m_{E}^{2}$ and $m_{L}^{2}$ :

$$
\begin{aligned}
& m_{E}^{2}=m_{E, 0}^{2}+c_{E 1} M_{1}^{2}+\tilde{c}_{E U} m_{U, 0}^{2}-\frac{1}{11} D^{2}\left(1-\frac{g_{1}^{2}}{g_{1,0}^{2}}\right)+\delta_{E, y_{\tau}}^{2} \\
& m_{L}^{2}=m_{L, 0}^{2}+c_{L 1} M_{1}^{2}+c_{L 2} M_{2}^{2}+\tilde{c}_{L Q} m_{Q, 0}^{2}+\frac{1}{22} D^{2}\left(1-\frac{g_{1}^{2}}{g_{1,0}^{2}}\right)+\delta_{L, y_{\tau}}^{2}
\end{aligned}
$$

where by $m_{S, 0}^{2}$ (with an additional index 0) for $S=E, L, Q, U, D$ we denote sfermion masses at the high scale, while $M_{1,2}$ are low-scale $\mathrm{U}(1)$ and $\mathrm{SU}(2)$ gaugino soft mass parameters. The coefficients $c_{E 1}$ and $c_{L i}$ can be found by solving the 1-loop RGEs, whereas $\tilde{c}_{E U i}, \tilde{c}_{L Q}$ by solving the 2-loop RGEs and identifying the leading effects; they are given in table 1 for some representative choices for the high scale $Q$ and the scale $m_{S}=\sqrt{m_{\tilde{t}_{1}} m_{\tilde{t}_{2}}}$ at which electroweak symmetry breaking is evaluated. ${ }^{2} D^{2}$ (denoted in literature also as $\mathcal{S}_{0}$ ) is defined as

$$
D^{2}=\mathcal{S}_{0}=\operatorname{tr}\left[Y \mathbf{M}_{\mathrm{scalars}, 0}^{2}\right]=m_{H_{u}}^{2}-m_{H_{d}}^{2}+\operatorname{tr}\left[\mathbf{m}_{Q, 0}^{2}-2 \mathbf{m}_{U, 0}^{2}+\mathbf{m}_{D, 0}^{2}-\mathbf{m}_{L, 0}^{2}+\mathbf{m}_{E, 0}^{2}\right]
$$

where $\mathbf{m}_{S, 0}^{2}$ are the $3 \times 3$ sfermion mass matrices at the high scale, $m_{H_{u}}^{2}$ and $m_{H_{u}}^{2}$ are the soft supersymmetry breaking masses of the Higgs doublets at the high scale, and $g_{1}\left(g_{1,0}\right)$

\footnotetext{
${ }^{2}$ We assume that at the high scales the soft supersymmetry breaking parameters are the same for all three generations; beyond that framework, e.g. in models with inverted hierarchy of soft supersymmetry breaking masses, two-loop contributions proportional to squark masses can drive $m_{L}^{2}$ to values smaller than $m_{E}^{2}$, opening up a possibility for yet another example of sneutrino LOSP [25].
} 
is the $\mathrm{U}(1)_{Y}$ gauge coupling at the low (high) scale. Leading corrections arising due to the $\tau$ Yukawa couplings are denoted by $\delta_{E, y_{\tau}}^{2}$ and $\delta_{L, y_{\tau}}^{2}$; for small and moderate values of $\tan \beta$ they are small and their only role is to make the third generation of sleptons slightly lighter than the first two, but they can become important if the mass parameters $\sqrt{m_{H_{d}}^{2}}$ at the high scale or the coefficient $A_{\tau}$ in the trilinear coupling of staus are much larger than $\sqrt{m_{L}^{2}}$ and $\sqrt{m_{E}^{2}}$. In the case when the colored particles are much heavier than the sleptons, as is usually the case, one should in principle include the leading two-loop contributions to the RGEs in order to obtain $\mathcal{O}(10 \mathrm{GeV})$ accuracy in mass determination.

Substituting (2.4) and (2.5) into (2.3), we see that the sneutrino can be the LOSP in two (mutually not exclusive) cases. Note first that in models with $D^{2}=0$ and universal gaugino masses, such as the CMSSM or the NUHM1 model (i.e. the NUHM with $m_{H_{u}}=m_{H_{d}}$ ), for which $M_{2} \approx 2 M_{1}$, and a high scale $>10^{14} \mathrm{GeV}$, the sneutrino cannot be the LOSP, since it is always heavier than the bino. We can then firstly demand $D^{2}<0$, which gives $m_{L}^{2}<M_{1}^{2}$, and then the sign difference in the coefficients multiplying $D^{2}$ in (2.4) and (2.5) can lead to $m_{L}^{2}<m_{E}^{2}$. This possibility is realized in the general NUHM and later we shall also discuss the corresponding mass spectra in some detail. The second option is to relax the gaugino mass universality. This possibility is naturally realized in GGM models, leading to the sneutrino LOSP and we shall later present some representative examples of mass spectra arising in such models, as well. ${ }^{3}$

\subsection{Higgs boson mass of $126 \mathrm{GeV}$}

Recent data from the LHC $[20,21]$ strongly suggest that the lightest Higgs boson has a mass of approximately $126 \mathrm{GeV}$. As we mentioned in the Introduction, this implies a larger supersymmetry breaking scale and implies non-trivial consequences for the possibility of having sneutrino NLSP with gravitino LSP.

At one loop, the lightest Higgs boson mass can be approximated as [26]

$$
m_{h}^{2} \approx m_{Z}^{2} \cos 2 \beta+\frac{3}{4 \pi^{2}} \frac{m_{t}^{4}}{v^{2}}\left[\log \frac{m_{S}^{2}}{m_{t}^{2}}+\frac{X_{t}^{2}}{m_{S}^{2}}\left(1-\frac{X_{t}^{2}}{12 m_{S}^{2}}\right)\right]
$$

where $v=174 \mathrm{GeV}, m_{S}^{2}$ is the (defined above) product of the stop masses and $X_{t}=$ $A_{t}-\mu / \tan \beta$. It is well known (see e.g. [27-31]) that consistency with the Higgs boson mass measurement at $\sim 126 \mathrm{GeV}$ points toward large values of $m_{S} \gtrsim \mathcal{O}(1) \mathrm{TeV}$ and values of $X_{t}$ maximizing the second term in the square bracket in (2.7), with largest values achieved for $X_{t} \sim \pm \sqrt{6} m_{S}$. (The other option of increasing $m_{S}$ so that the logarithmic correction in (2.7) gives the whole necessary contribution is less natural as it requires very heavy

\footnotetext{
${ }^{3}$ Another way would be to assume large $m_{Q, 0}$, since it would give a negative contribution to $m_{L}^{2}$. However, this would lead to large $\mu$, hence would increase the left-right mixing in the stau sector and would thus make the lighter stau lighter than the sneutrino.
} 


\begin{tabular}{|c|ccccccc|}
\hline coefficient & $c_{A}^{A_{t}}$ & $c_{1 / 2}^{A_{t}}$ & $c_{A}^{\mu}$ & $c_{A, 1 / 2}^{\mu}$ & $c_{1 / 2}^{\mu}$ & $c_{1 / 2}^{Q}$ & $c_{1 / 2}^{U}$ \\
\hline range & $\sim 0.4$ & $\sim 2$ & $\sim 0.04$ & $\sim 0.1$ & $\sim 3$ & $\sim 3$ & $1-2$ \\
\hline
\end{tabular}

Table 2. Approximate values of the coefficients $c_{\beta}^{\alpha}$ in (2.8)-(2.11) for $m_{S}$ varying from 1 to $5 \mathrm{TeV}$ and two patterns of gaugino masses at the high scale $Q=2 \times 10^{16} \mathrm{GeV}$ : universal gaugino case and $M_{1,0}, M_{2,0} \ll M_{3,0} \equiv m_{1 / 2}$.

stops.) A solution of the one-loop MSSM RGEs gives [24]:

$$
\begin{aligned}
A_{t} & =c_{A}^{A_{t}} A_{t, 0}-c_{1 / 2}^{A_{t}} m_{1 / 2} \\
\mu^{2} & \approx 3 c_{A}^{\mu} A_{t, 0}^{2}-3 c_{A, 1 / 2}^{\mu} A_{t, 0} m_{1 / 2}+c_{1 / 2}^{\mu} m_{1 / 2}^{2}+\ldots \\
m_{Q}^{2} & \approx-c_{A}^{\mu} A_{t, 0}^{2}+c_{A, 1 / 2}^{\mu} A_{t, 0} m_{1 / 2}+c_{1 / 2}^{Q} m_{1 / 2}^{2}+\ldots \\
m_{U}^{2} & \approx-2 c_{A}^{\mu} A_{t, 0}^{2}+2 c_{A, 1 / 2}^{\mu} A_{t, 0} m_{1 / 2}+c_{1 / 2}^{U} m_{1 / 2}^{2}+\ldots
\end{aligned}
$$

At one loop the values of the numerical coefficients $c_{\beta}^{\alpha}$ can be expressed as functions of the gauge and top Yukawa couplings. In table 2 we indicate typical values of these coefficients for different choices of $M_{S}$ and gaugino mass patterns.

For brevity, in (2.9)-(2.11) only the terms depending on the high-scale parameters $m_{1 / 2}$ and $A_{t, 0}$ are shown, as they suffice for the following argument. From (2.8)-(2.9) it is obvious that the easiest way of obtaining a large negative $X_{t}$ is to make the gluino rather heavy; increasing $A_{t, 0}$ by an equal amount is about five times less effective and may threaten to make the stops tachyonic. However, it should be kept in mind that a large $m_{1 / 2}$ tends to make $|\mu|$ large; it is of no particular consequence for $X_{t}$, as $\mu$ enters this quantity multiplied by $1 / \tan \beta$, but a large $|\mu|$ additionally increases left-right sfermion mixing, which, as we discussed in section 2.1 tends to make charged sleptons lighter than sneutrinos (for fixed sfermion masses at the high scale).

If the soft supersymmetry breaking mass parameters $A_{t}, m_{Q}^{2}$ and $m_{U}^{2}$ are dominated by the RGE contributions from the gluino mass, one obtains $X_{t} / m_{S} \approx X_{t} / \sqrt{m_{Q} m_{U}} \sim-1$, which is not very close to the maximal stop mixing scenario [32], optimal for a large Higgs boson mass (the second term in the square bracket in (2.7) is $\sim 2$ times smaller than its maximal value). A Higgs boson mass of $126 \mathrm{GeV}$ can be then obtained either by assuming a rather large $m_{1 / 2}$, or by taking a large negative $A_{t, 0}$, preferably $A_{t, 0} \sim-(1-3) m_{1 / 2}$ [33], or else by admitting tachyonic stops at high scales [30], which we shall not pursue further here.

We are therefore led to the conclusion that a Higgs boson mass of $126 \mathrm{GeV}$ puts an important constraint on the possibility of sneutrino LOSP by implying a higher scale of supersymmetry breaking. Lower bounds from direct SUSY searches are consistent with this trend but currently not yet as strong. A large Higgs boson mass favors large negative $A_{t}$ which is usually correlated with large $A_{\tau}$ via RG running; this increases left-right mixing in the charged slepton sector and makes a stau lighter than the sneutrino. Such a large $A_{t}$ most easily originates from a large $m_{1 / 2}$ (or a combination of slightly smaller $m_{1 / 2}$ and a large negative $A_{t, 0}$ ), which increases $\mu$, thereby also increasing left-right mixing in the charged slepton sector. In the following we will illustrate within two SUSY models employing different supersymmetry breaking mechanisms, and both allowing sneutrino LOSP, how 
a large value of $m_{1 / 2}$ implied by a heavy Higgs boson leads to strong constraints on the reheating temperature resulting from $\mathrm{BBN}$ bounds.

\subsection{Bound on the reheating temperature from BBN}

For gravitinos with a mass significantly smaller than the Fermi scale, their present abundance resulting from scatterings in thermal plasma [34-36] can be approximated by [37]:

$$
\Omega_{\tilde{G}}^{\mathrm{TP}} h^{2} \approx\left(\frac{T_{\mathrm{R}}}{10^{8} \mathrm{GeV}}\right)\left(\frac{1 \mathrm{GeV}}{m_{\tilde{G}}}\right) \sum_{r=1}^{3} \gamma_{r}\left(\frac{M_{r}}{900 \mathrm{GeV}}\right)^{2}
$$

where $m_{\tilde{G}}$ is the gravitino mass, $M_{r}$ denote gaugino mass parameters at the low scale and the coefficients $\gamma_{r}$ can be calculated from 1-loop RGEs for the gaugino masses and gauge couplings: they can be evaluated for $T_{\mathrm{R}}=10^{9}\left(10^{7}\right) \mathrm{GeV}$ as $\gamma_{3}=0.50(0.67), \gamma_{2}=$ $0.51(0.49), \gamma_{1}=0.20(0.15)$, for the gluino masses of $900 \mathrm{GeV}$. It is easy to read from this estimate that with $m_{1 / 2} \sim 1 \mathrm{TeV}$ and $m_{\tilde{G}}=100 \mathrm{GeV}$ the observed dark matter abundance implies a reheating temperature of $T_{R} \sim 5 \times 10^{8} \mathrm{GeV}$, which is close to minimal values $\sim 2 \times 10^{9} \mathrm{GeV}\left(\sim 2 \times 10^{8} \mathrm{GeV}\right)$ required by simple models of thermal leptogenesis with zero (thermal) initial abundance of the lightest right-handed neutrinos and sneutrinos [38, 39].

The lifetime of sneutrino LOSP can be approximated as

$$
\tau_{\mathrm{NLSP}}=\left(5.9 \times 10^{4} \mathrm{sec}\right)\left(\frac{m_{\tilde{G}}}{1 \mathrm{GeV}}\right)^{2}\left(\frac{100 \mathrm{GeV}}{m_{\mathrm{NLSP}}}\right)^{5}\left(1-\frac{m_{\tilde{G}}^{2}}{m_{\mathrm{NLSP}}^{2}}\right)^{-4}
$$

which can easily be of the order of $10^{5}-10^{7}$ sec. For such long lifetimes it is then possible that hadro-dissociation processes induced by a subdominant decay process of sneutrino LOSP where a quark-antiquark pair is produced can alter the BBN predictions beyond the current observational uncertainties. We shall study this issue in the following section, by numerically analyzing representative examples in two models of supersymmetry breaking which allow for sneutrino LOSP.

\section{$3 \quad$ Numerical analysis}

As we have argued in section 2.2, models of supersymmetry breaking at the high scale allow a sneutrino LOSP only if at least one of the two conditions: $D^{2}=0$ or $M_{1}: M_{2}: M_{3}=$ $\alpha_{1}: \alpha_{2}: \alpha_{3}$ is violated at the high scale. (At 1 loop these conditions are renormalization group invariants, hence they can be evaluated at any scale.) A violation of the former is manifest in the NUHM while the latter condition can be satisfied in many ways. A recently considered scenario is the GGM where it is assumed that the hidden and the messenger sectors in models of gauge mediation can be more complicated than what is required in a minimal theory. (The soft supersymmetry breaking parameters in both models are given in the appendix.) 
All scans: $m_{H_{u}}=500 \mathrm{GeV}, m_{H_{d}}=4000 \mathrm{GeV}, \mu>0$

\begin{tabular}{|c|cc|cc|}
\hline Case & \multicolumn{2}{|c|}{ varied parameters } & \multicolumn{2}{|c|}{ fixed parameters } \\
\hline 1 & $m_{0}$ & $m_{1 / 2}$ & $A_{0}=-3000 \mathrm{GeV}$ & $\tan \beta=10$ \\
2 & $A_{0}$ & $m_{1 / 2}$ & $m_{0}=300 \mathrm{GeV}$ & $\tan \beta=10$ \\
3 & $A_{0}$ & $\tan \beta$ & $m_{0}=300 \mathrm{GeV}$ & $m_{1 / 2}=1200 \mathrm{GeV}$ \\
4 & $m_{1 / 2}$ & $\tan \beta$ & $m_{0}=300 \mathrm{GeV}$ & $A_{0}=-3000 \mathrm{GeV}$ \\
\hline
\end{tabular}

Table 3. Description of scans over the parameters in the NUHM presented in figures 1 and 2.

\subsection{The NUHM}

Armed with the above analytical considerations, we will now identify regions of the NUHM parameter space where the sneutrino is the LOSP. We will determine if these solutions are consistent with the Higgs mass, low-energy observables and early Universe. The details of the scans are given in table 3. In our numerical work we used suspect [40] to solve the renormalization group equations and calculate mass spectra, micrOMEGAs [41] for the LOSP relic abundance and SuperIso [42] for flavor observables.

In figures 1 and 2 we present the LOSP identity and its mass, as well as the mass of the Higgs boson. In both panels of figure 1 and in the right panel of figure 2 the sneutrino LOSP region is bounded from above at large enough values of $m_{1 / 2}$. This can be easily understood since $m_{L}^{2}$ is a much faster growing function of $m_{1 / 2}$ than $M_{1}^{2}$ which is the bino mass squared. At fixed $m_{1 / 2}$ and increasing $m_{0}$, sfermion masses grow and they eventually become larger than the bino mass, which explains the bending of the boundary between the sneutrino and bino LOSP regions in the left panel of figure 1. A negative contribution to $m_{L}^{2}$, which is proportional to $D^{2}$, has to be overcome by some other positive contributions proportional to $m_{0}$ or $m_{1 / 2}$; otherwise we find unphysical regions (marked white) with tachyonic sleptons. It should also be mentioned that the negative contribution to $m_{U}^{2}$ (proportional to $D^{2}$ ) is larger by a factor of $3 / 2$ than that to $m_{L}^{2}$; the former parameter also receives a much larger renormalization group correction proportional to $m_{1 / 2}^{2}$ than the latter. As a consequence, for sufficiently small values of $m_{1 / 2}$ the lighter stop becomes lighter than the sleptons; the corresponding region of stop LOSP is visible in the left panel of figure 1. All these effects leads to a lower bound on $m_{1 / 2}$; in our scans we find no sneutrino LOSP models for $m_{1 / 2}<800 \mathrm{GeV}$, which, as we shall discuss later, has the important consequences for the maximum reheating temperature. As it can be seen in the right panels of figures 1 and 2 , for $\mu>0$ large negative values of $A_{0}$ result in large off-diagonal entries in the stop mass matrix and lead to very light and even tachyonic stops. The appearance of the bino LOSP region in figure 2 results from the $\tau$-Yukawa effect in the renormalization group equations: in the leading logarithm approximation, the quantities $\delta_{E, y_{\text {tau }}}^{2}$ in $(2.4)$ and $\delta_{L, y_{\tau}}^{2}$ in $(2.5)$ can be approximated by

$$
\delta_{E, y_{\tau}}^{2} \approx 2 \delta_{l, y_{\text {tau }}}^{2} \approx-\frac{1}{4 \pi^{2}} y_{\tau}^{2}\left(m_{H_{d}}^{2}+A_{0}^{2}\right) \log \left(\frac{M_{\mathrm{GUT}}}{m_{S}}\right),
$$

where $M_{\mathrm{GUT}}$ is the unification scale at which $m_{H_{d}}$ and $y_{\tau}$ are evaluated here. As $y_{\tau}$ 

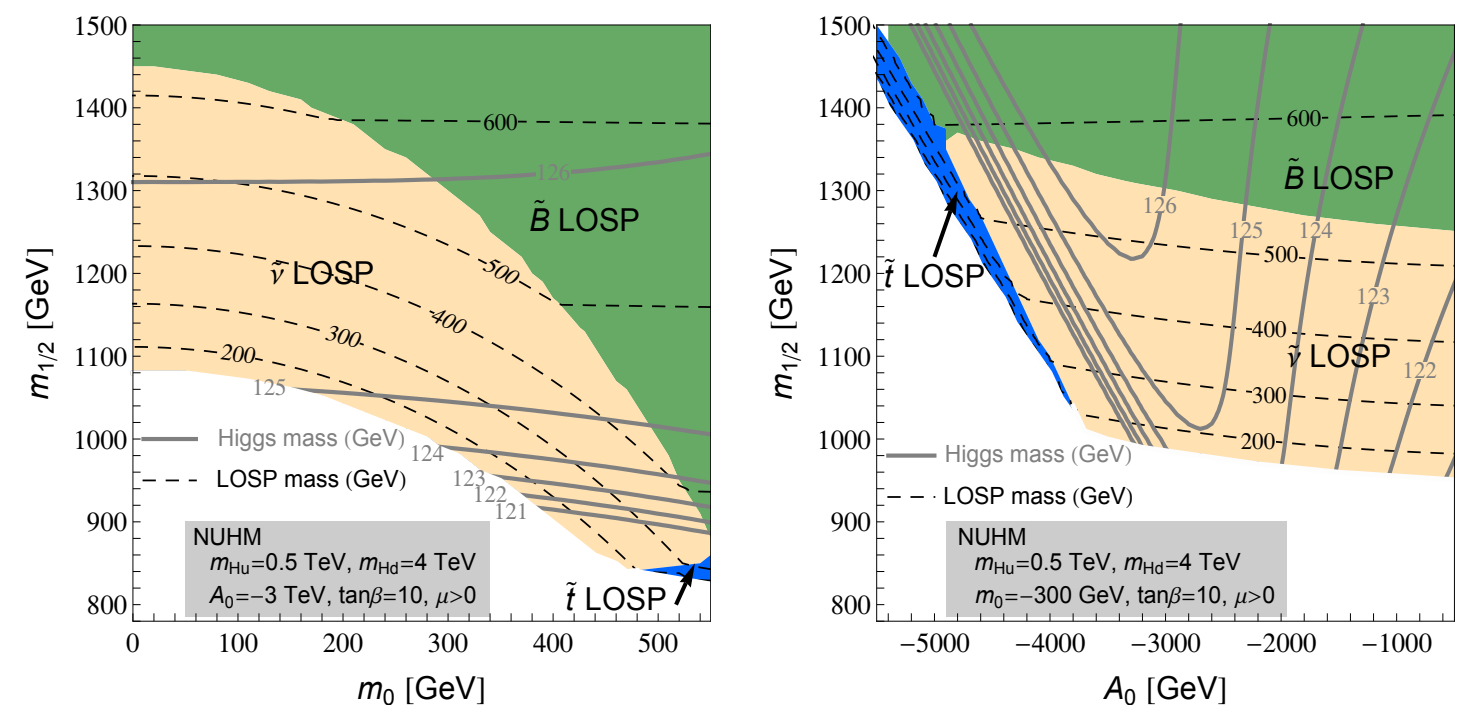

Figure 1. Slices of the NUHM parameter space: $m_{0}$ vs $m_{1 / 2}$ (left panel) and $A_{0}$ vs $m_{1 / 2}$ (right panel) with the values of $m_{H_{u}}=500 \mathrm{GeV}, m_{H_{d}}=4000 \mathrm{GeV}$ fixed at the unification scale and $\tan \beta=10, \mu>0$. Contours of constant LOSP (Higgs boson) masses are shown as dashed (solid) lines. Unphysical regions are marked in white.
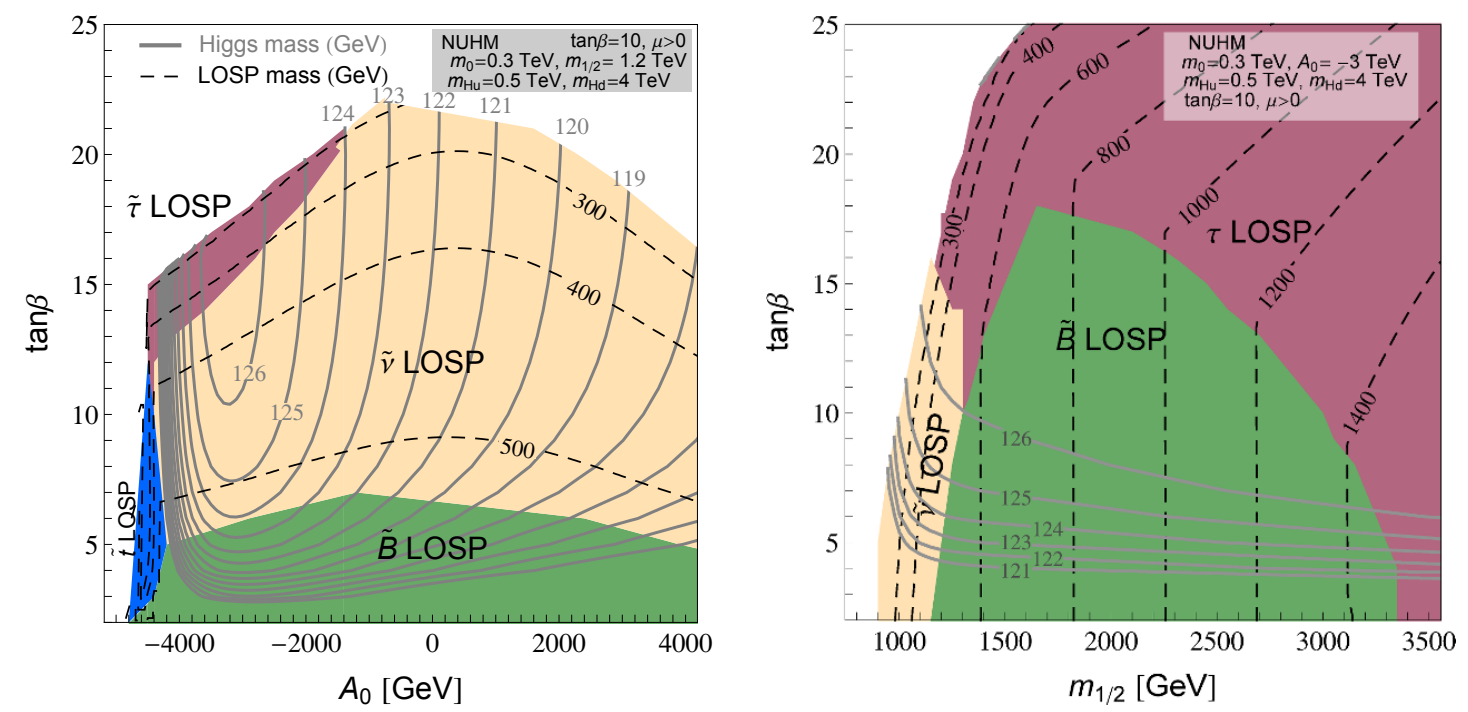

Figure 2. Slices of the NUHM parameter space: $A_{0}$ vs $\tan \beta$ (left panel) and $m_{1 / 2}$ vs $\tan \beta$ (right panel) with the values of $m_{0}=300 \mathrm{GeV}, m_{H_{u}}=500 \mathrm{GeV}, m_{H_{d}}=4000 \mathrm{GeV}$ fixed at the unification scale and $\mu>0$. Contours of constant LOSP (Higgs boson) masses are shown as dashed (solid) lines. Unphysical regions are marked in white.

increases with growing $\tan \beta$, we see that moderate values of $\tan \beta$ actually help one of the sleptons to become the LOSP.

Unsurprisingly, for fixed $A_{0}=-3000 \mathrm{GeV}$, we find a lower bound $m_{1 / 2} \gtrsim 1 \mathrm{TeV}$ resulting from the requirement that the Higgs boson mass exceeds $122 \mathrm{GeV}$, the value that we adopt as a conservative lower bound on the observable. For fixed $m_{0}$, the shape of the 


\begin{tabular}{|c|cccc|}
\hline observable & $\mathrm{BR}(b \rightarrow s \gamma)[45]$ & $\mathrm{BR}\left(B_{u} \rightarrow \tau \nu_{\tau}\right)[46]$ & $\mathrm{BR}\left(B_{s} \rightarrow \mu^{+} \mu^{-}\right)[47]$ & $\Delta M_{B_{s}}[48]$ \\
\hline lower bound & $2.8 \times 10^{-4}$ & $0.7 \times 10^{-4}$ & $0.7 \times 10^{-9}$ & $12.9 \mathrm{ps}^{-1}$ \\
upper bound & $4 \times 10^{-4}$ & $2.7 \times 10^{-4}$ & $6.3 \times 10^{-9}$ & $22.5 \mathrm{ps}^{-1}$ \\
\hline
\end{tabular}

Table 4. 95\% CL bounds for selected low-energy flavor and electromagnetic observables. Both experimental and theoretical errors have been taken into account.

\begin{tabular}{|c|cccc|}
\hline observable & $\mathrm{D} / \mathrm{H}$ & ${ }^{3} \mathrm{He} / \mathrm{D}$ & $Y_{\mathrm{p}}$ & ${ }^{6} \mathrm{Li} /{ }^{7} \mathrm{Li}$ \\
\hline lower bound & $1.2 \times 10^{-5}$ & & not applied & \\
upper bounds & $4 \times 10^{-5} / 5.3 \times 10^{-5}$ & 1.5 & 0.26 & $0.1 / 0.66$ \\
& & & & (stringent/conservative) \\
\hline
\end{tabular}

Table 5. 95\% CL BBN bounds based on [22]. The observables are ratios of the element abundances, with the obvious exception of $Y_{\mathrm{p}}$ which is ${ }^{4} \mathrm{He}$ mass fraction. The lower limit on $Y_{p}$ is irrelevant for constraining the abundance of decaying particles. The upper limit on $\mathrm{D} / \mathrm{H}$ represents a compromise between the commonly used average of the best determinations of this quantity and the large spread of the individual results. The use of two bounds for ${ }^{6} \mathrm{Li} /{ }^{7} \mathrm{Li}$, a stringent and a conservative one, reflects the uncertainty in estimating the efficiency of production/destruction of this element in stellar environment.

constant Higgs boson mass contours in the $\left(A_{0}, m_{1 / 2}\right)$ plane agrees with the requirements for maximal stop mixing [33]. For all the points shown in figures 1 and 2 the low-energy observables lie within a conservative $95 \%$ CL range quoted in table 4 . As in many unified models, the supersymmetric contribution to $\delta a_{\mu}$ is too low to explain the observed anomaly [43]. We also checked that for all the points of interest squark masses of the first and second generations are well above $1400 \mathrm{GeV}$, required by the LHC data [44]. In the stop LOSP regions, stop masses are often much smaller that $\sim 450 \mathrm{GeV}$ which is the lower limit from the LHC, but these regions are disfavored anyway, because the Higgs boson mass drops below $120 \mathrm{GeV}$ there.

For the region with sneutrino LOSP shown in the left panel of figure 1 we calculate the abundances of light elements following the method outlined in [22] and apply the observational limits shown in table 5. A representative sample of our results is shown in figures 3 and 4 . We find no constraints for the gravitino masses smaller than $7.5 \mathrm{GeV}$. At $m_{\tilde{G}}=10 \mathrm{GeV}$ a part of the parameter space corresponding to $m_{\tilde{\nu}} \gtrsim 500 \mathrm{GeV}$ or, equivalently, to $\tau_{\tilde{\nu}} \gtrsim 10^{3} \mathrm{~s}$, is excluded because of too large $\mathrm{D} / \mathrm{H}$ abundance. For all values of $m_{\tilde{G}}$ the bounds from ${ }^{6} \mathrm{Li} /{ }^{7} \mathrm{Li}$ are always more stringent than the $\mathrm{D} / \mathrm{H}$ bounds. A further increase of $m_{\tilde{G}}$ does not change this picture much, until the gravitino becomes degenerate with the sneutrino, which introduces a strong phase-space enhancement of the sneutrino lifetime. This is illustrated in the case with $m_{\tilde{G}}=250 \mathrm{GeV}$, for which the BBN bounds, while still dominated by ${ }^{6} \mathrm{Li} /{ }^{7} \mathrm{Li}$, become weaker.

A closer look at the actual predictions for $\mathrm{D} / \mathrm{H}$ in the considered parameter range of the NUHM reveals that even at points consistent with the allowed bounds, the abundance of $\mathrm{D}$ is altered with respect to the standard $\mathrm{BBN}$ value. It is also quite sensitive to the hadronic energy release: if we approximated it as $\left(m_{\tilde{\nu}}-m_{\tilde{G}}\right) / 3$, as is often done in the 

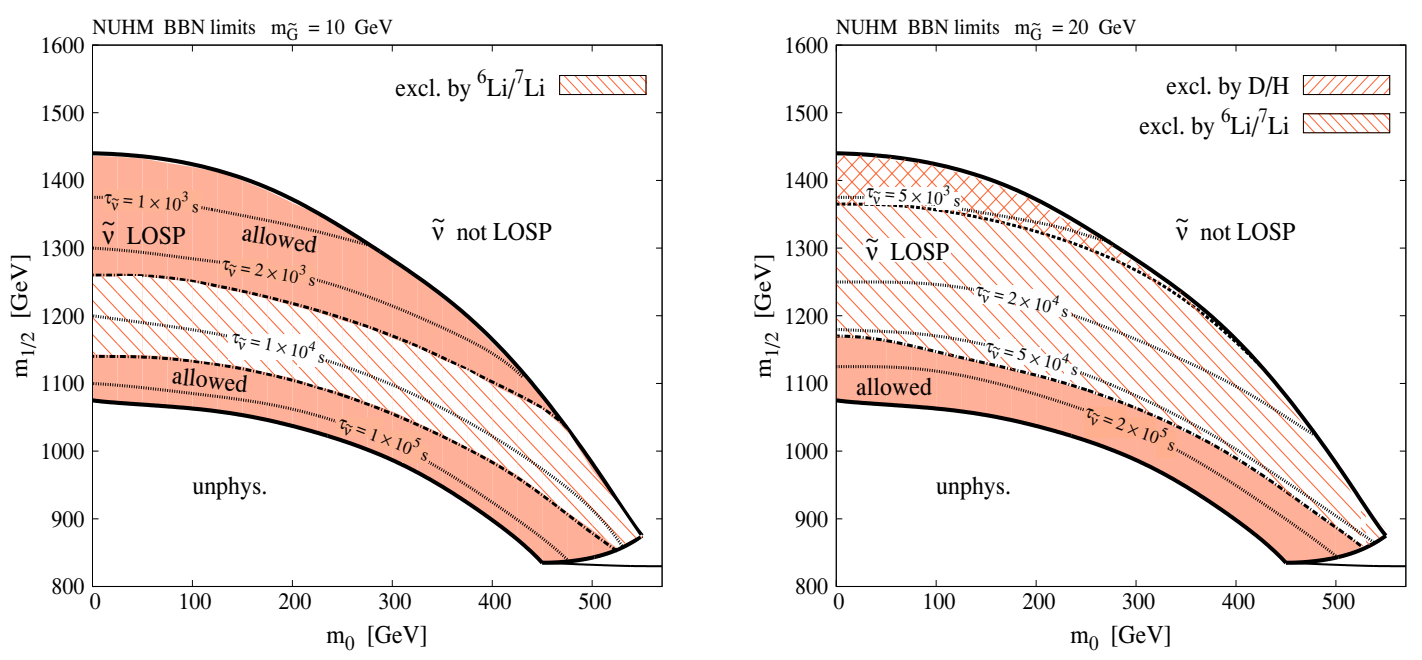

Figure 3. BBN bounds for the sneutrino LOSP region in the NUHM shown in the left panel of figure 1 for the values of gravitino mass of $m_{\tilde{G}}=10$ and $20 \mathrm{GeV}$. For ${ }^{6} \mathrm{Li} /{ }^{7} \mathrm{Li}$ the stringent limit was used; the conservative limit does not constrain the parameter space.
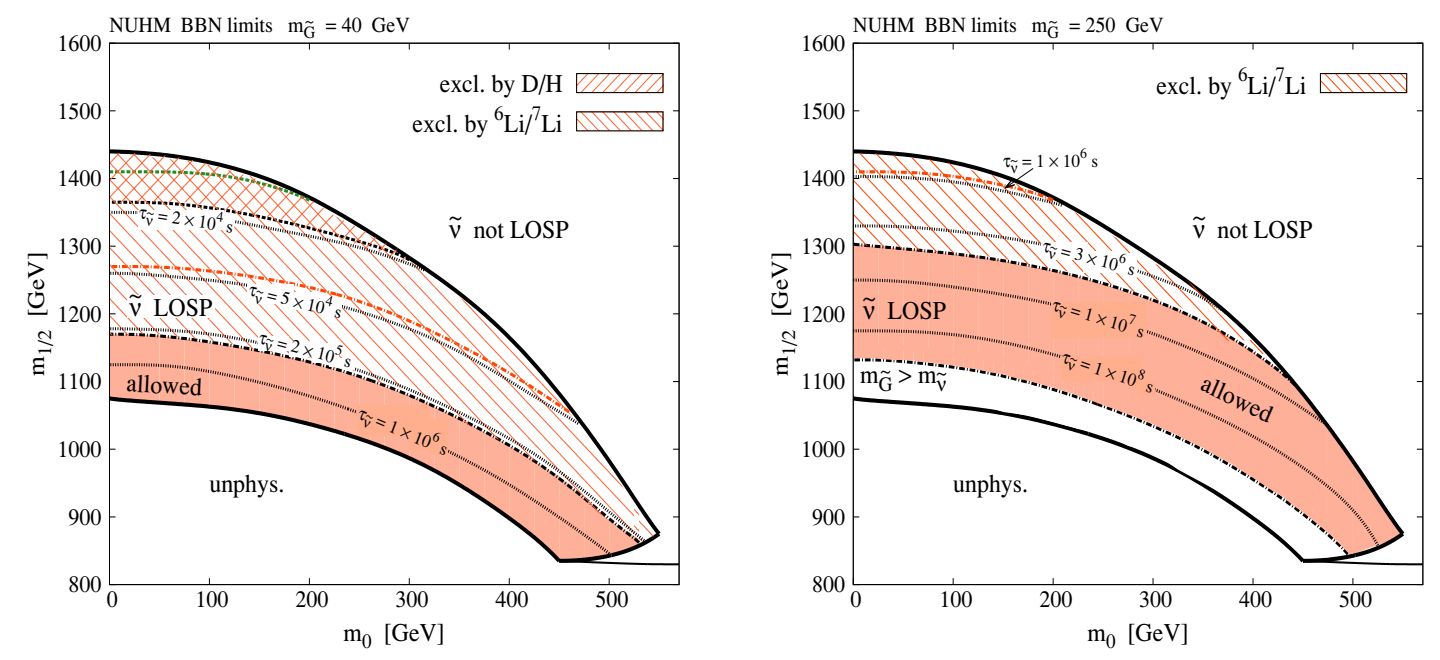

Figure 4. BBN bounds for the sneutrino LOSP region in the NUHM shown in the left panel of figure 1 for the values of gravitino mass of $m_{\tilde{G}}=40$ and $250 \mathrm{GeV}$. For ${ }^{6} \mathrm{Li} /{ }^{7} \mathrm{Li}$ the stringent limit was used; the boundary of the excluded region with the more conservative constraint for ${ }^{6} \mathrm{Li} /{ }^{7} \mathrm{Li}$ is represented by a red dash-dotted line. The dotted green line in the left panel shows the change in the lower boundary of the region excluded by $\mathrm{D} / \mathrm{H}$ if a more conservative limit $5.3 \times 10^{-5}$ is used [22].

literature, instead of calculating the energy of the $q \bar{q}$ pair produced in the sneutrino decay, then with the conservative ${ }^{6} \mathrm{Li} /{ }^{7} \mathrm{Li}$ limit the lower boundary of the respective excluded region in the right panel of figure 3 would shift downwards by as much as $\sim 100 \mathrm{GeV}$ (see the right panel of figure 5). In other words, one would significantly underestimate the sneutrino LOSP region allowed by the constraint. 

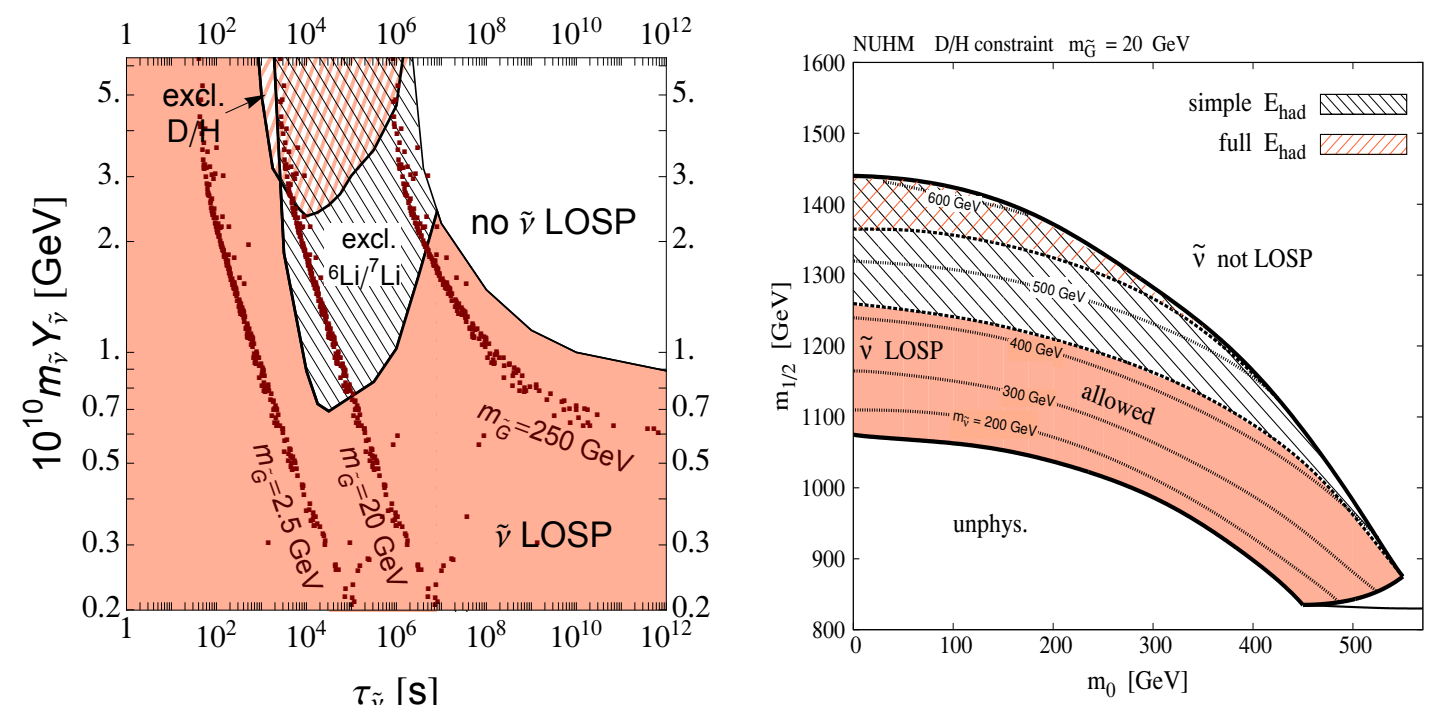

Figure 5. Left panel: BBN constraints shown in the $\tau_{\tilde{\nu}}$ vs $m_{\tilde{\nu}} Y_{\tilde{\nu}}$ plane for the sneutrino LOSP region shown in the left panel of figure 1 . Dots show the results of our scan with fixed $m_{\tilde{G}}=2.5$, 20 and $250 \mathrm{GeV}$. Right panel: the impact of different estimates of hadronic energy release on the $\mathrm{D} / \mathrm{H}$ bounds for $m_{\tilde{G}}=20 \mathrm{GeV}$. For the excluded region marked 'simple Ehad' an approximation $E_{\text {had }}=\left(m_{\tilde{\nu}}-m_{\tilde{G}}\right) / 3$ was used, while the excluded region marked 'full Ehad' corresponds to a computation of $E_{\text {had }}$ involving integration over the full 4-body phase space.

In order to understand better the origin of the BBN constraints, we first project all the analyzed points onto the $\tau_{\tilde{\nu}}$ vs $m_{\tilde{\nu}} Y_{\tilde{\nu}}$ plane; this is shown in the left panel of figure 5 . We also show there the bounds from the abundances of those light elements that constrain parameter space regions with sneutrino LOSP. Since $\Omega_{\tilde{\nu}} h^{2}$ is roughly proportional to $m_{\tilde{\nu}}^{2}$ (neglecting the opening of additional annihilation channels for increased $\tilde{\nu}$ masses) and since for $m_{\tilde{G}} \ll m_{\tilde{\nu}}$ the sneutrino lifetime scales as $\tau_{\tilde{\nu}} \propto m_{\tilde{G}}^{2} m_{\tilde{\nu}}^{-5}$, it is easy to understand why, with increasing $m_{\tilde{G}}$, the constraints from $\mathrm{D} / \mathrm{H}$ and ${ }^{6} \mathrm{Li} /{ }^{7} \mathrm{Li}$ first appear, next tighten up and then eventually become weaker. As can be also easily seen from (2.13), a partial degeneracy between $m_{\tilde{G}}$ and $m_{\tilde{\nu}}$ causes a much larger increase of $\tau_{\tilde{\nu}}$ than the simple power law above implies, hence the BBN bounds become correspondingly weaker. Those features can easily be seen for the results of our scan with a three values of fixed gravitino mass of $m_{\tilde{G}}=2.5,20,250 \mathrm{GeV}$, as a band of dark red dots.

One may worry that for long sneutrino lifetimes, $\tau_{\tilde{\nu}}>10^{7} \mathrm{~s}$, the electromagnetic showers produced in scatterings of energetic neutrinos from sneutrino decays off neutrinos of cosmic background can affect the BBN by altering the ${ }^{3} \mathrm{He} / \mathrm{H}$ abundance $[9,49]$. In order to verify this we determined that the exclusion plots from [9] do not provide additional constraints on our parameter space; we also interpolated the exclusion plots from [49] in $\left(m_{\tilde{\nu}}, \tau_{\tilde{\nu}}, B_{h}\right)$ plane and found no significant impact from ${ }^{3} \mathrm{He} / \mathrm{D}$.

As we have seen, increasing $m_{\tilde{G}}$ for a given $m_{\tilde{\nu}}$ tends to alleviate the BBN constraints. However, for large gravitino masses there is another factor that we have to take into account. Non-thermal gravitinos produced in sneutrino LOSP decays will have velocities much larger than those characteristic for thermal distribution. Such fast moving dark matter particles 

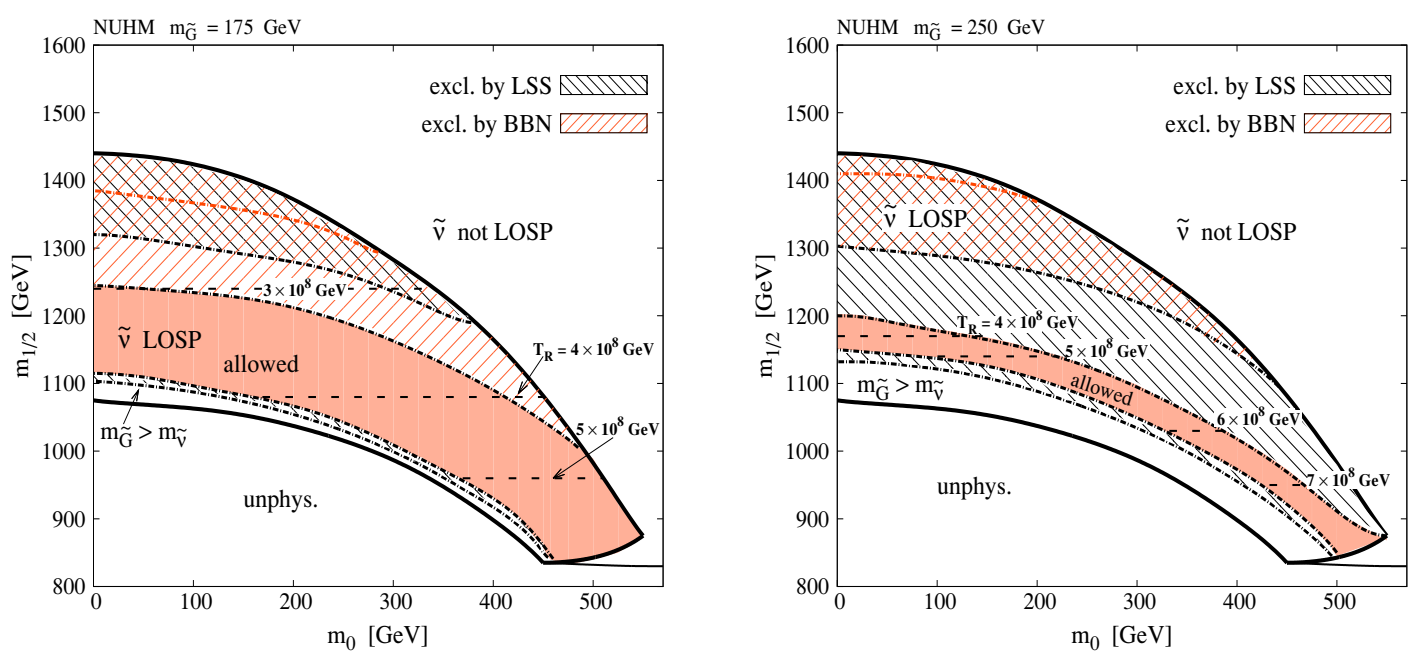

Figure 6. A comparison of the BBN and LSS bounds for $m_{\tilde{G}}=175$ and $250 \mathrm{GeV}$. Long-dashed lines show contours of constant reheating temperature $T_{R}$. For ${ }^{6} \mathrm{Li} /{ }^{7} \mathrm{Li}$ the stringent limit was used; the boundary of the excluded region with the more conservative constraint for ${ }^{6} \mathrm{Li} /{ }^{7} \mathrm{Li}$ is represented by a red dash-dotted line.

tend to erase small scales of Large Scale Structures (LSS), especially when they constitute a sizable fraction of the dark matter density. Following [50], we account for these LSS constraints by requiring that the root mean square velocity of the non-thermally produced dark matter gravitinos does not exceed $1 \mathrm{~km} / \mathrm{s}$ and that the non-thermal component makes less than $20 \%$ of the total dark matter abundance.

The impact of this bound on the NUHM parameter space is shown in figure 6, where we show the superposition of the BBN bound discussed previously and the above LSS bounds for $m_{\tilde{G}}=175$ and $250 \mathrm{GeV}$. At such large $m_{\tilde{G}}$, the LSS bounds become more stringent than the BBN ones (at $m_{\tilde{\nu}} \geq 300 \mathrm{GeV}$ the LOSP relic abundance, $\Omega_{\mathrm{LOSP}} h^{2}$, exceeds $20 \%$ of the total dark matter abundance, hence $\Omega_{\tilde{G}}^{\mathrm{NTP}} h^{2}$ is also of this order), leaving just a small allowed strip in the parameter space. For $m_{\tilde{G}}>270 \mathrm{GeV}$, we find that the LSS bounds exclude the entire section of the parameter space that we analyze here. This has important consequences for the maximum reheating temperature, since limits on the maximum reheating temperature become weaker with increasing gravitino mass.

A summary of our results is presented in figure 7 which shows regions in the $\left(m_{\tilde{G}}, m_{\tilde{\nu}}\right)$ plane excluded by our constraints. It is clear that the BBN bounds alone allow two distinct regions in the parameter space. For small $m_{\tilde{G}}<10 \mathrm{GeV}$, there are no constraints on $m_{\tilde{\nu}}$ but the allowed maximum reheating temperature is relatively low, $T_{R}^{\max } \sim 10^{7} \mathrm{GeV}$. For larger $m_{\tilde{G}}$, the BBN bounds start constraining the sneutrino mass and the maximum reheating temperature increases to $\sim 10^{9} \mathrm{GeV}$ when $m_{\tilde{\nu}} \sim m_{\tilde{G}}$. Imposing the LSS bounds closes this second region, thus slightly reducing the reheating temperature down to $\sim 9 \times 10^{8} \mathrm{GeV}$. However, now the points for which $T_{R}$ is maximal correspond to Higgs boson masses much smaller than the LHC measurement. The requirement that the Higgs boson mass is at least $122 \mathrm{GeV}$, brings $T_{R}^{\max }$ down to $7 \times 10^{8} \mathrm{GeV}$. 


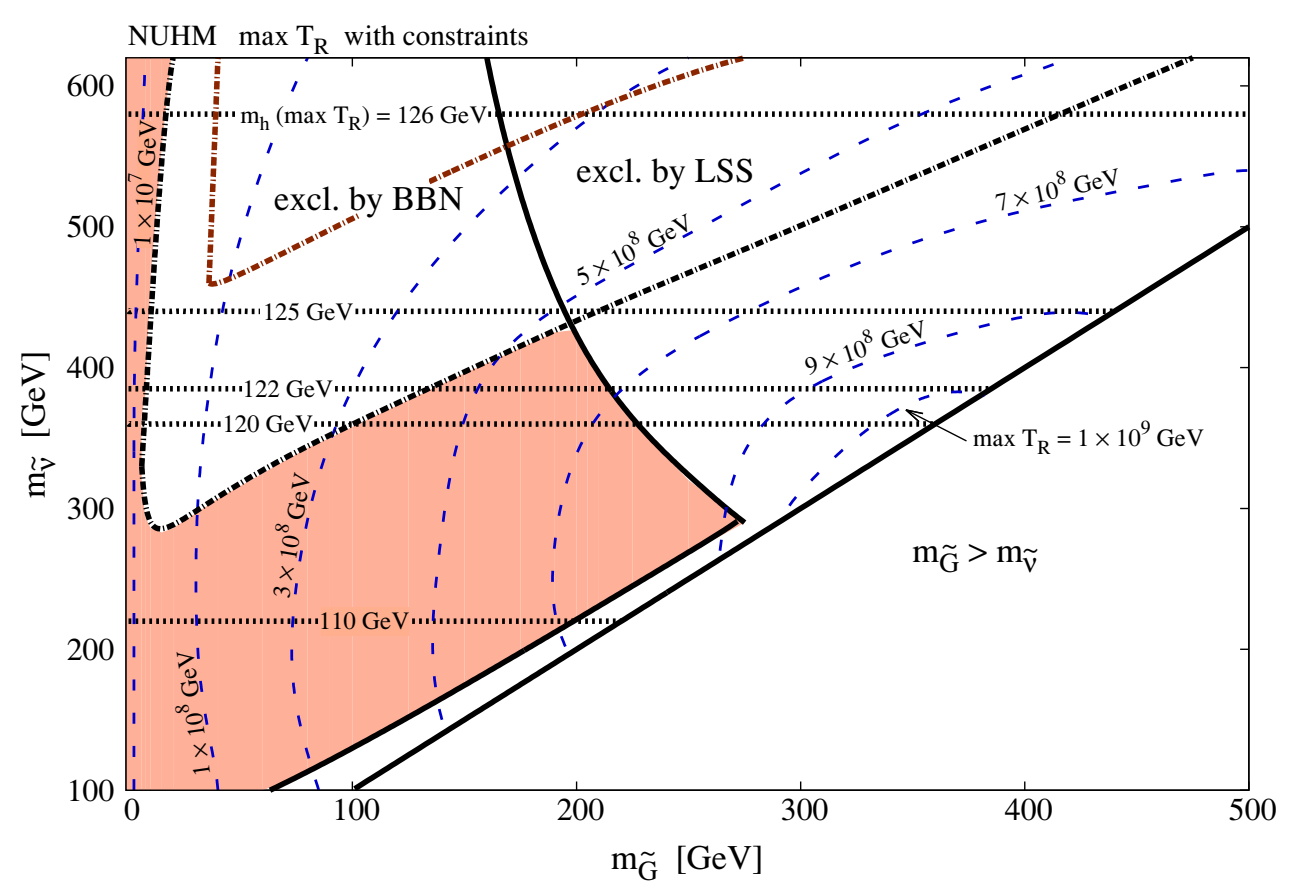

Figure 7. A summary of the bounds in the NUHM in the $\left(m_{\tilde{G}}, m_{\tilde{\nu}}\right)$ plane. The thick dashed line bounds the region excluded by BBN, the solid red line marks the boundary of the region excluded by LSS. Thinner dashed lines show the maximum reheating temperature, $T_{R}^{\max }$, and thinner dotted lines show the Higgs boson mass corresponding to $T_{R}^{\max }$. For ${ }^{6} \mathrm{Li} /{ }^{7} \mathrm{Li}$ the stringent limit was used; the boundary of the excluded region with the more conservative constraint for ${ }^{6} \mathrm{Li} /{ }^{7} \mathrm{Li}$ is represented by a red dash-dotted line.

These bounds on maximum $T_{R}$ as a function of $m_{\tilde{G}}$ are shown in the left panel of figure 8 for the same sets of constraints. In the panel we impose the BBN bounds and we show the results with and without the LSS bounds and with and without the requirement that the Higgs boson mass is at least $122 \mathrm{GeV}$. We see that in each case the maximum $T_{R}$ lies close to $10^{9} \mathrm{GeV}$, depending on the set of bounds imposed. Without the LSS or the Higgs boson mass bounds, this constraint mainly results from the lower bound on $m_{1 / 2}$, as the maximum $T_{R}$ scales roughly as $m_{1 / 2}^{-2}$. This can be seen in the right panel of figure 8 where we show the maximum $T_{R}$ versus the Higgs boson mass with and without BBN and LSS constraints. Note that at $m_{h}=126 \mathrm{GeV}$ the maximum $T_{R}$ plunges down as the BBN and the LSS bound become inconsistent with larger values of the Higgs boson mass.

These maximum values of $T_{R}$ is close to the quoted above lower bound required by simple thermal leptogenesis. It should be noted that the quoted leptogenesis bounds should be treated as indicative rather than absolute, since a rather mild mass degeneracy in the right-handed neutrino sector may lower the minimum reheating temperature for successful leptogenesis by a factor of a few [51]. 

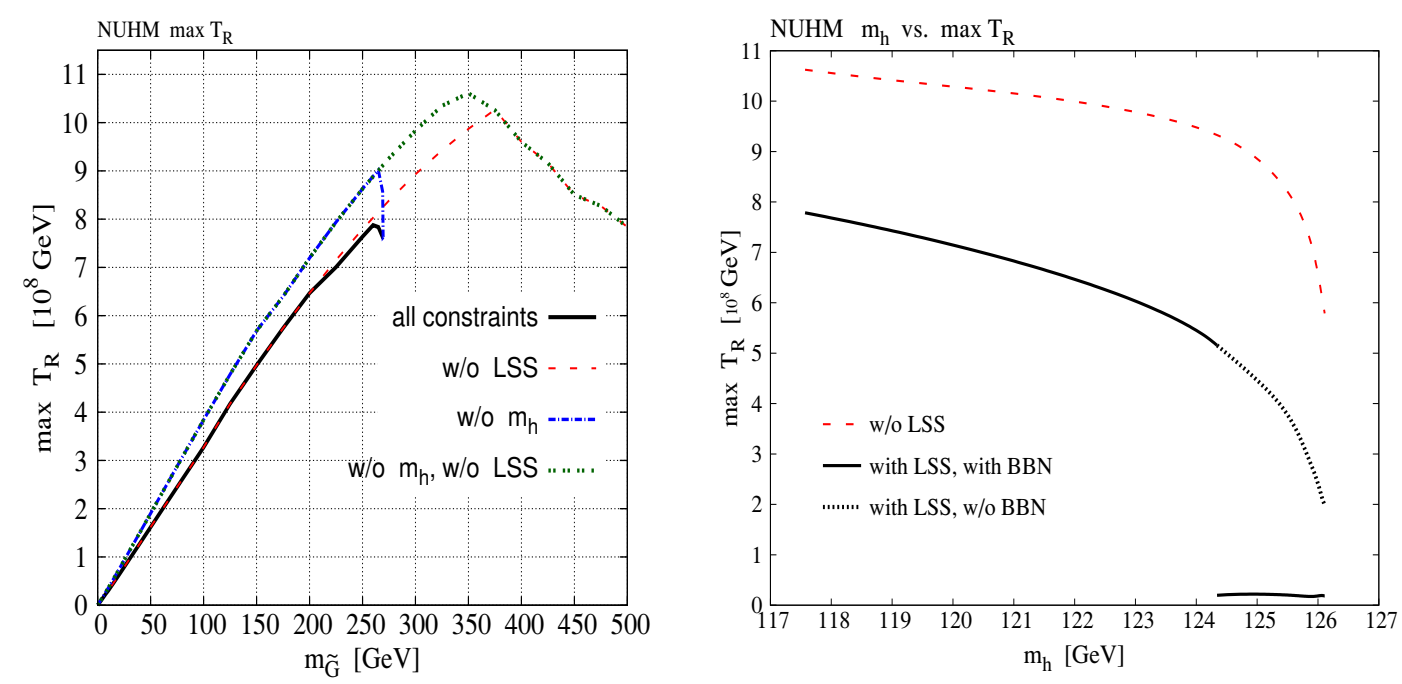

Figure 8. Left panel: the maximum reheating temperature as a function of $m_{\tilde{G}}$ with the BBN, the LSS and the Higgs boson mass constraints $\left(m_{h}>122 \mathrm{GeV}\right)$ applied, as well as without one or both of the LSS and the Higgs boson mass constraints. Right panel: maximum reheating temperature versus the Higgs boson mass without the LSS constraint (upper red dashed line) and with the LSS constraint (lower line). The solid (dotted) segments correspond to the cases where the BBN bound is (is not) applied.

\subsection{GGM models}

Another class of theoretically motivated scenarios in which it is possible to obtain sneutrino LOSP are models of Generalized Gauge Mediation. Unlike in the NUHM, where the condition $\operatorname{tr}\left(Y \mathbf{M}_{\text {scalars }}^{2}\right)=0$ is violated, the feature of GGM models that allows for a sneutrino LOSP is a non-universality of the gaugino masses. In particular, it follows from (2.4) that sneutrino LOSP is viable for $M_{2} / M_{1} \lesssim 2$ at the electroweak scale. We shall therefore utilize the freedom of gaugino mass assignment offered by GGM models to reduce $M_{2,0}$ at the messenger scale without breaking the universality of the two remaining gauginos, i.e. we shall adopt $M_{1,0}=M_{3,0}$. More specifically, we shall assume $M_{1,0}: M_{2,0}: M_{3,0}=5: 2: 5$, which predicts that the lightest gaugino-like neutralino is a wino. Eqs. (2.4) also show that for sneutrino LOSP $m_{L}^{2}$ cannot be too large, which for fixed gaugino mass scale places upper bounds on the parameters $\tilde{\Lambda}_{1}$ and $\tilde{\Lambda}_{2}$, whose relation to scalar masses at the messenger scale is shown in the appendix. These bounds can be seen in the left panel of figure 9 , which also shows that increasing $\tilde{\Lambda}_{2}$ with fixed $\tilde{\Lambda}_{1}$ increases $m_{L, 0}^{2}$ with respect to $m_{E, 0}^{2}$, which may lead to a right-handed stau LOSP.

The interplay between the gaugino and scalar mass scales is shown in the right panel of figure 9 , where we keep $\tilde{\Lambda}_{1}=2 \tilde{\Lambda}_{2}$. Increasing gaugino masses while keeping the mass ratios fixed enlarges the range of $\tilde{\Lambda}_{2}$ (and $\tilde{\Lambda}_{1}$ ) for which one of the sleptons is the LOSP. With small values of $\tilde{\Lambda}_{1}$ slepton masses are governed by 1-loop corrections proportional to gaugino masses and, as follows from (2.4), right-handed staus are the lightest. By increasing $\tilde{\Lambda}_{2}$ one can obtain sneutrino LOSP, because the slepton masses at the messenger 

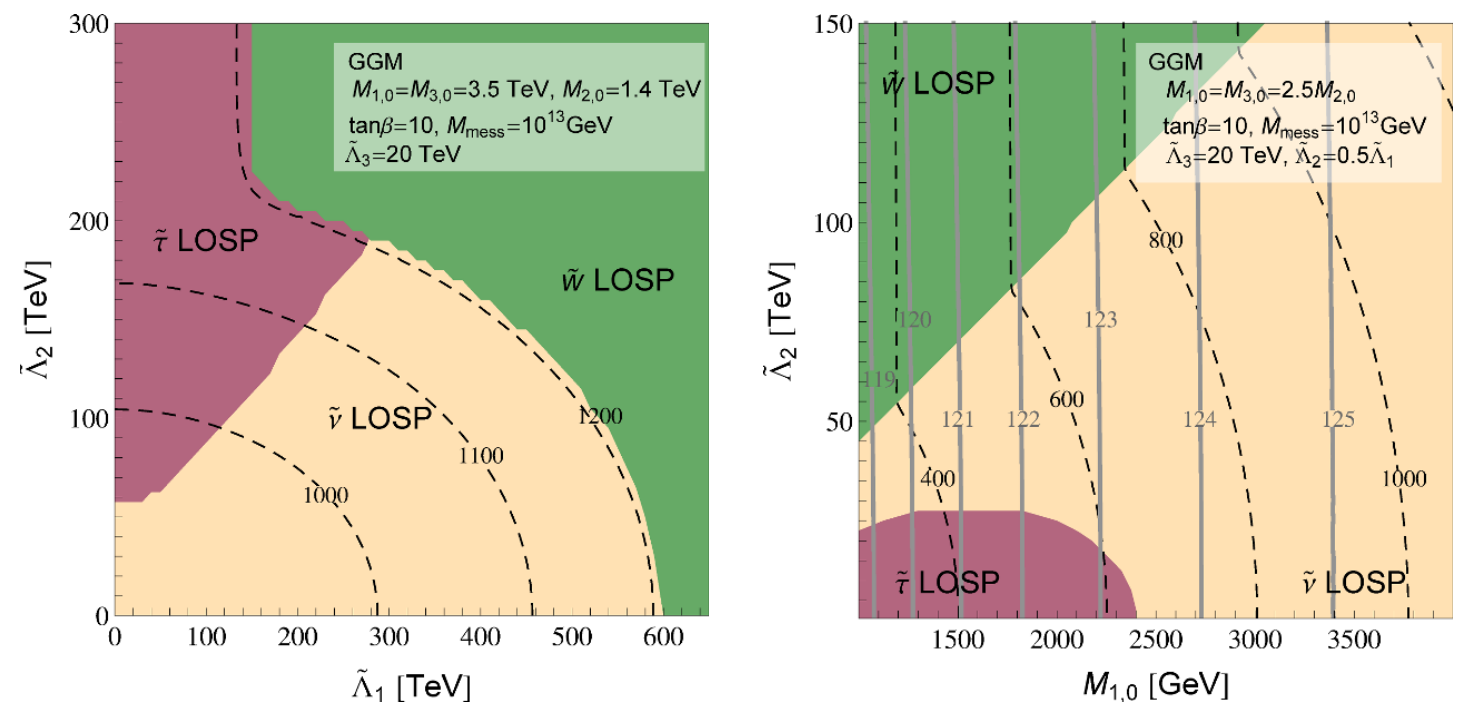

Figure 9. Sections of the GGM parameter space: $\tilde{\Lambda}_{1}$ vs $\tilde{\Lambda}_{2}$ (left panel) and $M_{1,0}$ vs $\tilde{\Lambda}_{2}$ (right panel) with fixed ratio $M_{1,0}: M_{2,0}: M_{3,0}=5: 2: 5$ and fixed values of $\tan \beta=10$, the messenger scale $M_{\text {mess }}=10^{13} \mathrm{GeV}$ and $\tilde{\Lambda}_{3}=20 \mathrm{TeV}$ with $\mu>0$. Contours of constant LOSP (Higgs boson) masses are shown as dashed (solid) lines.

scale, $m_{L, 0}^{2}$ and $m_{E, 0}^{2}$, contain contributions proportional to the product of $\tilde{\Lambda}_{1}^{2}$ and the square of the respective hypercharge, which is larger for right-handed sleptons.

GGM models do not allow $A$-terms at the messenger scale. (This can, however, be circumvented by adding direct messenger-matter couplings in the superpotential [52], consistently with a Higgs mass of $126 \mathrm{GeV}$ [53].) Therefore, in order to have a sufficiently large Higgs boson mass, we have to consider much larger gluino mass $M_{3,0}$ at the messenger scale, which generates radiatively large stop masses and a large negatively $A_{t}$ at the electroweak scale. The left panel of figure 9 corresponds to fixed gaugino mass parameters and, therefore, to an almost constant Higgs boson mass of $126 \mathrm{GeV}$; in the right panel, the Higgs boson mass becomes close to $126 \mathrm{GeV}$ for large values of $M_{1,0}=M_{3,0}$. At first sight it might seem that one could use the non-universality of the gaugino masses to make $M_{1,0}$ and $M_{2,0}$ much smaller than $M_{3,0}$, but a large $M_{3,0}$ also results in a large $\mu$ parameter at the electroweak scale, which prevents the sneutrino from being the LOSP. This is also the reason for adopting a relatively small value of $\tilde{\Lambda}_{3}$ : too large squark masses at the messenger scale also increase $\mu$. All this results in sneutrino LOSP masses of about $1 \mathrm{TeV}$, which is much larger than in the case of the NUHM.

Models with gauge mediation of supersymmetry breaking have the advantage that the leading contributions to the soft masses are flavor-diagonal, while the subdominant gravitymediated contributions, of the order of $m_{\tilde{G}}$, do not have to exhibit any such structure. This leads to a natural suppression of the FCNC's, but also has the obvious consequence that $m_{\tilde{G}} \ll m_{\tilde{\nu}}$, with the precise hierarchy depending on details of an appropriate flavor model. For this reason we do not consider $m_{\tilde{G}}$ larger than $20 \mathrm{GeV}$, hence the reheating temperature consistent with the measured dark matter abundance is much lower for GGM than 

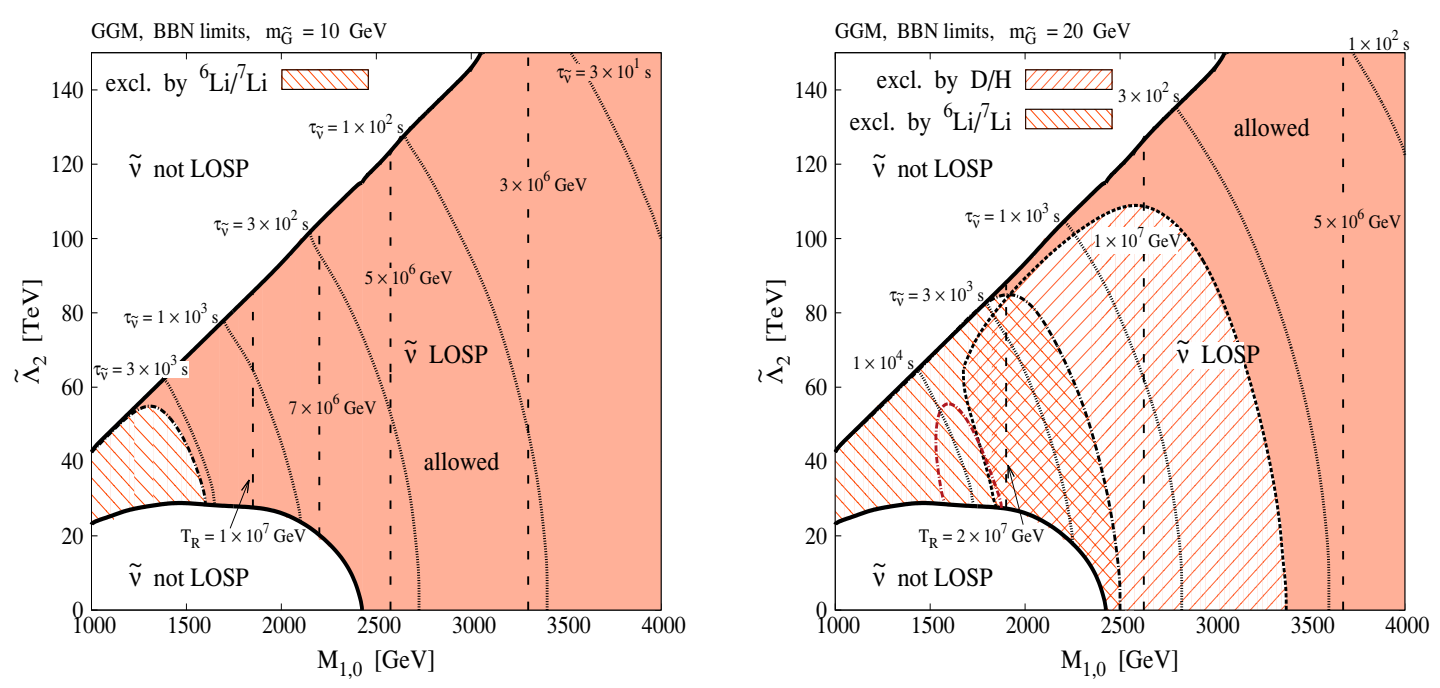

Figure 10. BBN bounds for the sneutrino LOSP region in GGM model shown in the right panel of figure 9 for values of gravitino mass $m_{\tilde{G}}=10$ and $20 \mathrm{GeV}$. For ${ }^{6} \mathrm{Li} /{ }^{7} \mathrm{Li}$ the stringent limit was used; the boundary of the excluded region with the more conservative constraint for ${ }^{6} \mathrm{Li} /{ }^{7} \mathrm{Li}$ is represented by a red dash-dotted line.

for the NUHM. Nonetheless, we find some BBN constraints for $m_{\tilde{G}} \sim \mathcal{O}(10) \mathrm{GeV}$; they are shown in figure 10.

\section{Conclusions}

In this paper, we have analyzed models of gravitino dark matter with the $\tau$-sneutrino as the lightest ordinary supersymmetric particle. We have shown that if the scale at which supersymmetry is broken is close to the unification scale, the sneutrino can be the LOSP either if gauginos are non-universal or $D^{2}<0$ at the high scale. We have then performed a detailed study of representative examples of these two possibilities: one arising in the NUHM and the other in models of generalized gauge mediation of supersymmetry breaking.

We have calculated the changes in the BBN predictions due to hadronic showers from sneutrino decays $\tilde{\nu} \rightarrow \nu \tilde{G} q \bar{q}$, calculating the hadronic energy release by a numerical integration over the phase space of the produced particles. In the cases in which the $\mathrm{D} / \mathrm{H}$ bound provided the strongest constraint, we observed big changes of the excluded regions of the parameter space between this calculation and one using a simplified formula for the hadronic energy release.

We showed that in models of gravitino dark matter with sneutrino LOSP, the four classes of constraints that can be applied: (i) the BBN constrains, (ii) the constraints on the large structure formation due to a presence of free-streaming decay products of $\tilde{\nu}$, (iii) the Higgs boson mass bounds derived from the LHC data and (iv) the bounds on reheating temperature required by simple models of thermal leptogenesis are inconsistent, albeit the maximum reheating temperature is only $2-3$ times smaller than the value suggested by the leptogenesis bound. (This was clearly visible in the NUHM; with gauge mediation, 
such large reheating temperatures were unattainable due to theoretical constraints on the gravitino mass.) Therefore, our results challenge the notion that models of gravitino dark matter with sneutrino LOSP are compatible with simple thermal leptogenesis.

\section{Acknowledgments}

This work has been funded in part by the Welcome Programme of the Foundation for Polish Science. LR is also supported in part by the Polish National Science Centre grant N N202 167440, an STFC consortium grant of Lancaster, Manchester and Sheffield Universities and by the EC 6th Framework Programme MRTN-CT-2006-035505. KT is partly supported by the MNiSW grants N N202 091839 and IP2011 056971.

\section{A Soft supersymmetry breaking parameters at the high scale}

Here we collect expressions for the soft supersymmetry breaking parameters at the high scale in the NUHM and the GGM model.

The parametrization of the NUHM is very simple

$$
\begin{aligned}
M_{1,0} & =M_{2,0}=M_{3,0}=m_{1 / 2} \\
m_{Q, 0}^{2} & =m_{U, 0}^{2}=m_{D, 0}^{2}=m_{L, 0}^{2}=m_{E, 0}^{2}=m_{0}^{2},
\end{aligned}
$$

while $m_{H_{u}}^{2}, m_{H_{d}}, A_{0}, \tan \beta$ and $\operatorname{sgn}(\mu)$ can are free parameters.

In GGM models, the soft supersymmetry breaking masses at the high scale in the notation of [19] read

$$
\begin{aligned}
M_{r, 0} & =\left(\alpha_{r} / 4 \pi\right) \Lambda_{1} \quad \text { for } r=1,2,3 \\
m_{Q, 0}^{2} & =(8 / 3)\left(\alpha_{3}^{2} / 16 \pi^{2}\right) \tilde{\Lambda}_{3}^{2}+(3 / 2)\left(\alpha_{2}^{2} / 16 \pi^{2}\right) \tilde{\Lambda}_{2}^{2}+(1 / 30)\left(\alpha_{1}^{2} / 16 \pi^{2}\right) \tilde{\Lambda}_{1}^{2} \\
m_{U, 0}^{2} & =(8 / 3)\left(\alpha_{3}^{2} / 16 \pi^{2}\right) \tilde{\Lambda}_{3}^{2}+(8 / 15)\left(\alpha_{1}^{2} / 16 \pi^{2}\right) \tilde{\Lambda}_{1}^{2} \\
m_{D, 0}^{2} & =(8 / 3)\left(\alpha_{3}^{2} / 16 \pi^{2}\right) \tilde{\Lambda}_{3}^{2}+(2 / 15)\left(\alpha_{1}^{2} / 16 \pi^{2}\right) \tilde{\Lambda}_{1}^{2} \\
m_{L, 0}^{2} & =m_{H_{u}}^{2}=m_{H_{d}}^{2}=(3 / 2)\left(\alpha_{2}^{2} / 16 \pi^{2}\right) \tilde{\Lambda}_{2}^{2}+(3 / 10)\left(\alpha_{1}^{2} / 16 \pi^{2}\right) \tilde{\Lambda}_{1}^{2} \\
m_{E, 0}^{2} & =(6 / 5)\left(\alpha_{1}^{2} / 16 \pi^{2}\right) \tilde{\Lambda}_{1}^{2} .
\end{aligned}
$$

The trilinear scalar couplings are all equal to zero and $\tan \beta, \operatorname{sgn}(\mu)$ are free parameters.

Open Access. This article is distributed under the terms of the Creative Commons Attribution License which permits any use, distribution and reproduction in any medium, provided the original author(s) and source are credited.

\section{References}

[1] S.P. Martin, A supersymmetry primer, hep-ph/9709356 [INSPIRE].

[2] L. Covi, J.E. Kim and L. Roszkowski, Axinos as cold dark matter, Phys. Rev. Lett. 82 (1999) 4180 [hep-ph/9905212] [INSPIRE]. 
[3] WMAP collaboration, E. Komatsu et al., Seven-Year Wilkinson Microwave Anisotropy Probe (WMAP) Observations: Cosmological Interpretation, Astrophys. J. Suppl. 192 (2011) 18 [arXiv:1001.4538] [INSPIRE].

[4] K.-Y. Choi, J.E. Kim, H.M. Lee and O. Seto, Neutralino dark matter from heavy axino decay, Phys. Rev. D 77 (2008) 123501 [arXiv:0801.0491] [INSPIRE].

[5] K. Jedamzik and M. Pospelov, Big Bang Nucleosynthesis and Particle Dark Matter, New J. Phys. 11 (2009) 105028 [arXiv:0906.2087] [InSPIRE].

[6] F. Iocco, G. Mangano, G. Miele, O. Pisanti and P.D. Serpico, Primordial Nucleosynthesis: from precision cosmology to fundamental physics, Phys. Rept. 472 (2009) 1 [arXiv: 0809.0631] [INSPIRE].

[7] M. Pospelov and J. Pradler, Big Bang Nucleosynthesis as a Probe of New Physics, Ann. Rev. Nucl. Part. Sci. 60 (2010) 539 [arXiv:1011.1054] [INSPIRE].

[8] T. Falk, K.A. Olive and M. Srednicki, Heavy sneutrinos as dark matter, Phys. Lett. B 339 (1994) 248 [hep-ph/9409270] [INSPIRE].

[9] M. Kawasaki and T. Moroi, Gravitino decay into a neutrino and a sneutrino in the inflationary universe, Phys. Lett. B 346 (1995) 27 [hep-ph/9408321] [INSPIRE].

[10] T. Kanzaki, M. Kawasaki, K. Kohri and T. Moroi, Cosmological constraints on gravitino LSP scenario with sneutrino NLSP, Phys. Rev. D 75 (2007) 025011 [hep-ph/0609246] [INSPIRE].

[11] L. Covi and S. Kraml, Collider signatures of gravitino dark matter with a sneutrino NLSP, JHEP 08 (2007) 015 [hep-ph/0703130] [INSPIRE].

[12] J.R. Ellis, K.A. Olive and Y. Santoso, Sneutrino NLSP scenarios in the NUHM with gravitino dark matter, JHEP 10 (2008) 005 [arXiv: 0807.3736] [INSPIRE].

[13] N. Polonsky and A. Pomarol, GUT effects in the soft supersymmetry breaking terms, Phys. Rev. Lett. 73 (1994) 2292 [hep-ph/9406224] [INSPIRE].

[14] D. Matalliotakis and H.P. Nilles, Implications of nonuniversality of soft terms in supersymmetric grand unified theories, Nucl. Phys. B 435 (1995) 115 [hep-ph/9407251] [INSPIRE].

[15] M. Olechowski and S. Pokorski, Electroweak symmetry breaking with nonuniversal scalar soft terms and large tan beta solutions, Phys. Lett. B 344 (1995) 201 [hep-ph/9407404] [INSPIRE].

[16] D. Shih, Spontaneous R-symmetry breaking in O'Raifeartaigh models, JHEP 02 (2008) 091 [hep-th/0703196] [INSPIRE].

[17] C. Cheung, A.L. Fitzpatrick and D. Shih, (Extra)ordinary gauge mediation, JHEP 07 (2008) 054 [arXiv:0710.3585] [INSPIRE].

[18] P. Meade, N. Seiberg and D. Shih, General Gauge Mediation, Prog. Theor. Phys. Suppl. 177 (2009) 143 [arXiv:0801.3278] [INSPIRE].

[19] L.M. Carpenter, M. Dine, G. Festuccia and J.D. Mason, Implementing General Gauge Mediation, Phys. Rev. D 79 (2009) 035002 [arXiv: 0805. 2944] [INSPIRE].

[20] ATLAS collaboration, Observation of a new particle in the search for the Standard Model Higgs boson with the ATLAS detector at the LHC, Phys. Lett. B 716 (2012) 1 [arXiv: 1207.7214] [INSPIRE]. 
[21] CMS collaboration, Observation of a new boson at a mass of $125 \mathrm{GeV}$ with the CMS experiment at the LHC, Phys. Lett. B $\mathbf{7 1 6}$ (2012) 30 [arXiv:1207.7235] [INSPIRE].

[22] K. Jedamzik, Big bang nucleosynthesis constraints on hadronically and electromagnetically decaying relic neutral particles, Phys. Rev. D 74 (2006) 103509 [hep-ph/0604251] [INSPIRE].

[23] H.E. Haber and G.L. Kane, The Search for Supersymmetry: Probing Physics Beyond the Standard Model, Phys. Rept. 117 (1985) 75 [InSPIRE].

[24] M.S. Carena, P.H. Chankowski, M. Olechowski, S. Pokorski and C. Wagner, Bottom-up approach and supersymmetry breaking, Nucl. Phys. B 491 (1997) 103 [hep-ph/9612261] [INSPIRE].

[25] M. Badziak, private communication.

[26] H.E. Haber, R. Hempfling and A.H. Hoang, Approximating the radiatively corrected Higgs mass in the minimal supersymmetric model, Z. Phys. C 75 (1997) 539 [hep-ph/9609331] [INSPIRE].

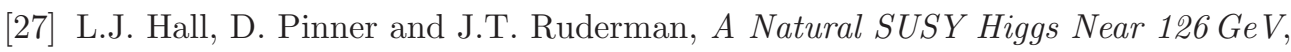
JHEP 04 (2012) 131 [arXiv:1112.2703] [INSPIRE].

[28] S. Heinemeyer, O. Stal and G. Weiglein, Interpreting the LHC Higgs Search Results in the MSSM, Phys. Lett. B 710 (2012) 201 [arXiv:1112.3026] [INSPIRE].

[29] A. Arbey, M. Battaglia and F. Mahmoudi, Constraints on the MSSM from the Higgs Sector: A pMSSM Study of Higgs Searches, $B_{s}^{0} \rightarrow \mu^{+} \mu^{-}$and Dark Matter Direct Detection, Eur. Phys. J. C 72 (2012) 1906 [arXiv:1112.3032] [INSPIRE].

[30] P. Draper, P. Meade, M. Reece and D. Shih, Implications of a $125 \mathrm{GeV}$ Higgs for the MSSM and Low-Scale SUSY Breaking, Phys. Rev. D 85 (2012) 095007 [arXiv:1112.3068] [INSPIRE].

[31] M. Carena, S. Gori, N.R. Shah and C.E. Wagner, A $125 \mathrm{GeV}$ SM-like Higgs in the MSSM and the $\gamma \gamma$ rate, JHEP 03 (2012) 014 [arXiv:1112.3336] [INSPIRE].

[32] R. Dermisek and H.D. Kim, Radiatively generated maximal mixing scenario for the Higgs mass and the least fine tuned minimal supersymmetric standard model, Phys. Rev. Lett. 96 (2006) 211803 [hep-ph/0601036] [INSPIRE].

[33] F. Brummer, S. Kraml and S. Kulkarni, Anatomy of maximal stop mixing in the MSSM, JHEP 08 (2012) 089 [arXiv: 1204.5977] [INSPIRE].

[34] M. Bolz, A. Brandenburg and W. Buchmüller, Thermal production of gravitinos, Nucl. Phys. B 606 (2001) 518 [Erratum ibid. B 790 (2008) 336-337] [hep-ph/0012052] [INSPIRE].

[35] J. Pradler and F.D. Steffen, Thermal gravitino production and collider tests of leptogenesis, Phys. Rev. D 75 (2007) 023509 [hep-ph/0608344] [INSPIRE].

[36] V.S. Rychkov and A. Strumia, Thermal production of gravitinos, Phys. Rev. D 75 (2007) 075011 [hep-ph/0701104] [INSPIRE].

[37] M. Olechowski, S. Pokorski, K. Turzynski and J.D. Wells, Reheating temperature and gauge mediation models of supersymmetry breaking, JHEP 12 (2009) 026 [arXiv:0908.2502] [INSPIRE]. 
[38] G. Giudice, A. Notari, M. Raidal, A. Riotto and A. Strumia, Towards a complete theory of thermal leptogenesis in the SM and MSSM, Nucl. Phys. B 685 (2004) 89 [hep-ph/0310123] [INSPIRE].

[39] S. Antusch and A. Teixeira, Towards constraints on the SUSY seesaw from flavour-dependent leptogenesis, JCAP 02 (2007) 024 [hep-ph/0611232] [INSPIRE].

[40] A. Djouadi, J.-L. Kneur and G. Moultaka, SuSpect: A Fortran code for the supersymmetric and Higgs particle spectrum in the MSSM, Comput. Phys. Commun. 176 (2007) 426 [hep-ph/0211331] [INSPIRE].

[41] G. Bélanger, F. Boudjema, A. Pukhov and A. Semenov, MicrOMEGAs 2.0: A Program to calculate the relic density of dark matter in a generic model, Comput. Phys. Commun. 176 (2007) 367 [hep-ph/0607059] [InSPIRE].

[42] F. Mahmoudi, SuperIso v2.3: A Program for calculating flavor physics observables in Supersymmetry, Comput. Phys. Commun. 180 (2009) 1579 [arXiv:0808.3144] [INSPIRE].

[43] M. Benayoun, P. David, L. DelBuono and F. Jegerlehner, An Update of the HLS Estimate of the Muon g-2, arXiv:1210.7184 [INSPIRE].

[44] Particle Data Group collaboration, O. Buchmueller and P. de Jong, rpp2012-rev-susy-2-experiment (2012).

[45] http://www.slac.stanford.edu/xorg/hfag/rare/2012/radll/index.html.

[46] Heavy Flavor Averaging Group collaboration, Y. Amhis et al., Averages of B-Hadron, C-Hadron and tau-lepton properties as of early 2012, arXiv:1207.1158 [INSPIRE].

[47] LHCB collaboration, First evidence for the decay $B_{s}^{0} \rightarrow \mu^{+} \mu^{-}$, Phys. Rev. Lett. 110 (2013) 021801 [arXiv:1211.2674] [INSPIRE].

[48] Particle Data Group collaboration, J. Beringer et al., Review of Particle Physics (RPP), Phys. Rev. D 86 (2012) 010001 [InSPIRE].

[49] T. Kanzaki, M. Kawasaki, K. Kohri and T. Moroi, Cosmological Constraints on Neutrino Injection, Phys. Rev. D 76 (2007) 105017 [arXiv:0705.1200] [INSPIRE].

[50] K. Jedamzik, M. Lemoine and G. Moultaka, Gravitino, axino, Kaluza-Klein graviton warm and mixed dark matter and reionisation, JCAP 07 (2006) 010 [astro-ph/0508141] [INSPIRE].

[51] S. Davidson, E. Nardi and Y. Nir, Leptogenesis, Phys. Rept. 466 (2008) 105 [arXiv:0802.2962] [INSPIRE].

[52] Z. Chacko and E. Ponton, Yukawa deflected gauge mediation, Phys. Rev. D 66 (2002) 095004 [hep-ph/0112190] [InSPIRE].

[53] T. Jelinski, J. Pawełczyk and K. Turzynski, On Low-Energy Predictions of Unification Models Inspired by F-theory, Phys. Lett. B 711 (2012) 307 [arXiv:1111.6492] [INSPIRE]. 